\title{
The influence of spinal position on imaging findings: an observational study of thoracolumbar spine upright MRI in elite gymnasts
}

\author{
Louise Fawcett ${ }^{1}\left[\right.$. Steven James ${ }^{2} \cdot$ Rajesh Botchu $^{2} \cdot$ James Martin $^{3} \cdot$ Nicola R. Heneghan ${ }^{4} \cdot$ Alison Rushton ${ }^{5,6}$
}

Received: 18 June 2021 / Revised: 18 June 2021 / Accepted: 14 September 2021 / Published online: 6 October 2021

(c) The Author(s) 2021

\begin{abstract}
Purpose To investigate whether upright magnetic resonance imaging (MRI) has a role in defining thoracolumbar spine pathology in elite gymnastics.

Methods A prospective cross-sectional observational study of National Senior and Junior Artistic gymnasts in three MRI positions (standard supine, upright flexed and extended positions). Two specialist musculoskeletal radiologists independently analysed images with neutral as a baseline with the effects of flexion and extension reported in line with Strengthening the Reporting of Observational Studies in Epidemiology (STROBE) statement.

Results Forty (18 males) gymnasts aged 13-24 years with a mean (SD) of 32 (5.3) training hours per week consented with $75 \%$ showing MRI abnormalities. Degenerative disc disease (DDD) was evident in 55\% participants with vertebral end plate (VEP) changes in $42.5 \%$. Spondylolysis was present in $40 \%$ with an additional $17 \%$ showing chronic bilateral complete L5 pars defects. 23\% participants demonstrated different MRI findings in upright flexion compared to neutral.

Conclusion Findings suggest a high levels of MRI abnormalities in elite gymnastics including altered disc morphology and posterior element abnormalities. High prevalence of T11/12 DDD and VEP changes reflects the thoracolumbar junction being a transition zone. Upright MRI and varying spine position offer promise for enhanced visualisation of posterior element abnormalities.
\end{abstract}

Keywords Lumbar spine $\cdot$ Elite gymnastics $\cdot$ Pathology $\cdot$ Upright MRI $\cdot$ Prospective

Louise Fawcett

louise.fawcett@eis2win.co.uk

1 British Gymnastics, English Institute of Sport, Lilleshall National Sports Centre, Nr Newport, Shropshire TF10 9AT, UK

2 Department of Radiology, School of Sport, Exercise and Rehabilitation Sciences, Royal Orthopaedic Hospital, Birmingham, University of Birmingham, West Midlands, UK

3 Institute of Applied Health Research, University of Birmingham, West Midlands, UK

4 Centre of Precision Rehabilitation for Spinal Pain (CPR Spine), School of Sport, Exercise and Rehabilitation Sciences, University of Birmingham, West Midlands, UK

5 School of Physical Therapy, Western University, London, Canada

6 Centre of Precision Rehabilitation for Spinal Pain, School of Sport, Exercise and Rehabilitation Sciences, University of Birmingham, West Midlands, UK

\section{Introduction}

The prevalence of low back pain (LBP) in gymnastics is high with reports of $85 \%$ in male and $65 \%$ in female artistic gymnastics [1]. The unique physical challenges of gymnastics with repetitive multi-directional movements with up to 13 times body weight on landing, place extreme stresses on the spine contributing to high rates of LBP [2]. Lumbar spine MRI studies of gymnasts have demonstrated a high prevalence of abnormalities, including degenerative disc degeneration, facet joint and posterior bony element stress changes [3].

Altered disc morphology is dependent on the type of components present (degeneration, vertebral endplate changes and herniations) to determine how tissues carry out load bearing and multi-directional movement [4]. Point prevalence estimates of degenerative disc disease (DDD) have ranged from $63 \%$ [3] to $75 \%$ [1] in gymnastic populations although the extent of the disc pathologies remains unknown. 
Research findings indicate athletic populations are predisposed to posterior element abnormality due to hormonal influences [5], and progression from pars interarticularis stress reaction through spondylolysis to spondylolisthesis is common in adolescent athletes [6]. Moreover, posterior element abnormalities are commonly observed at multiple thoracolumbar levels in cricketers [7]; however, studies involving elite gymnasts are scarce. Two MRI studies of elite female gymnasts found $16 \%$ in spondylolysis and $16 \%$ spondylolisthesis $(n=19)$ [2] and spondylolisthesis in $37 \%(n=8)$ [8]. One radiographical study reported low prevalence of spondylolysis (5.6\%) and no evidence of spondylolisthesis in female elite gymnasts $(n=18)$ [9]. Different imaging modalities may explain the variation in findings.

Standard supine lumbar MRI is widely considered the gold standard for investigating LBP, although the emergence of upright MRI offers capability to scan in a full flexed or extended spine offering the potential for visualisation of abnormal findings, meaning that it is different from normal or usual findings that are seen with conventional supine position [10]. It is not known whether upright MRI has a role in detailing altered disc morphology or posterior element abnormality of the thoracolumbar spine.

Knowledge of prevalence data regarding MRI abnormalities in elite gymnasts is important to aid understanding of potential injury mechanisms to use this data to inform the development of management strategies and approaches to training.

\section{Study aim}

To investigate thoracolumbar spine MRI findings in elite artistic gymnasts to:

\section{Objectives}

1. Establish the prevalence of altered disc morphology (e.g. DDD) and posterior element abnormalities (e.g. spondylolysis).

2. Investigate the influence of thoracolumbar spine position (standard supine, upright flexed, upright extended) on MRI findings.

\section{Methods}

\section{Study design}

A prospective cross-sectional upright MRI observational study involves senior musculoskeletal radiologists, musculoskeletal rehabilitation researchers and an experienced physiotherapist. The study is reported in accordance with the Strengthening the Reporting of Observational Studies in Epidemiology (STROBE) statement [11] (Appendix 1). The University of Birmingham Research Ethics committee granted approval for this study.

\section{Setting}

Data collection from consenting participants was at a single upright MRI imaging centre across two dates (May and October 2017).

\section{Participants and study size}

Inclusion criteria were a non-probability consecutive sample [12] of sixty-six elite male and female gymnasts, aged 12-26 within the British Gymnastics (BG) National Artistic Senior and Junior squads who were eligible for selection for the Tokyo Olympic Games in 2020.

\section{Variables and definitions}

DDD (DDD with anterior ring apophyseal injuries) was classified using the Pfirrmann grading of asymmetry in disc structure, distinction of nucleus and annulus, signal intensity and height [13]. Disc herniations were described as circumferential bulge ( $>50 \%$ disc circumference); broad-based herniation (25-50\% disc circumference); focal herniation ( $<25 \%$ disc circumference); and extrusion or sequestration [14]. Examining vertebral end plate (VEP) involved Modic et al.'s classification types 1-3 [15]. Spondylolysis was defined as stress reaction with bone marrow oedema with no fracture line in the pars interarticularis or pedicle, and spondylolisthesis as stress fracture and sliding of the vertebrae [16]. Facet joint arthropathy described as bone remodelling \pm facet joint effusions was classified using a grading system (0-3) with normal as grade 0 [17].

\section{Data collection and measurement}

MRI investigation was performed by a radiographer independent of the study team using a 0.5 Tesla MR Open system (Paramed, Genova, Italy) comprising sagittal T2 and STIR (short tau inversion recovery) and axial T2-weighted sequences (approximately 45'/participant). The three positions were standard supine (SS), upright flexed position (UFP) and upright extended position (UEP).

Images were interpreted on a standard PACS (picture archiving and communication system) workstation (Sectra, Sweden) reflecting normal clinical practice.

\section{Data analysis and bias considerations}

Two specialist musculoskeletal radiologists (SJ and RB) analysed the MRI data independently with a subsequent 
consensus discussion. No clinical data were available to radiologists. Detected abnormalities were categorised as follows:

- Altered disc morphology: DDD, vertebral end plate (VEP) changes and disc herniations.

- Posterior element abnormality: spondylolysis, spondylolisthesis, facet joint and any other abnormality.

Images were analysed for alteration in alignment at each vertebral level in the thoracolumbar spine, with differences in central and foraminal dimensions examined (in neutral as a baseline and then in flexion and extension). Participant demographics including gender, age and training hours were quantified with numbers, range and mean with standard deviation. Data analysis was conducted using SPSS, version 26.

\section{Results}

\section{Participant demographics and LBP characteristics}

Forty (male $n=18$ ) participants consented, aged 13-24 years, mean (SD): age 16.3 (2.7) engaged in 32 (5.3) training hours per week. Fifteen participants (37.5\%) reported experiencing current LBP and $33(82.5 \%)$ reported a history of LBP.

\section{Descriptive data}

\section{Presence of abnormalities in SS (Objective 1)}

Seventy-five per cent of participants (males $n=15$ aged 14-24, females $n=15$ aged 13-20) had abnormalities in the thoracolumbar spine in SS (Table 1).

\section{Altered disc morphology}

\section{DDD}

Twenty-two participants (55\%) showed evidence of DDD (Table 1). One spinal level was involved in 8 participants (36.4\%), two levels in 12 participants (54.5\%) and three levels in 2 participants (9\%). In total, 38 discs demonstrated DDD with the majority at T11/12 (23.7\%), followed by L5/ S1 (18.4\%) and T10/11 (15.8\%), with $42.1 \%$ of six other
Table 1 Presence of MRI abnormalities in SS

$n=40$

$n(\%)$

Altered disc morphology

Degenerative disc disease $22(55)$

Vertebral end plate changes $17(42.5)$

Disc herniations $8(20)$

Posterior element abnormalities

Pars oedema/spondylolysis $16(40)$

Spondylolisthesis $6(15)$

Facet joint $6(15)$

Other abnormalities

Left L5 perineural cyst, minor scoliosis in mid-lumbar 2 (5) region

Values presented are numbers (\%)

Some participants had multiple outcomes

Table 2 Spinal levels associated with altered disc morphology

\begin{tabular}{llll}
\hline Spinal levels & $\begin{array}{l}\text { DDD }(n=38) \\
n(\%)\end{array}$ & $\begin{array}{l}\text { VEP }(n=48) \\
n(\%)\end{array}$ & $\begin{array}{l}\text { Disc } \\
\text { herniations } \\
(n=11) \\
n(\%)\end{array}$ \\
\hline $\mathrm{T} 9 / 10$ & $0(0)$ & $0(0)$ & $0(0)$ \\
$\mathrm{T} 10 / 11$ & $6(15.8)$ & $11(22.9)$ & $0(0)$ \\
$\mathrm{T} 11 / 12$ & $9(23.7)$ & $14(29.2)$ & $0(0)$ \\
$\mathrm{T} 12 / \mathrm{L} 1$ & $5(13.2)$ & $9(18.7)$ & $1(9)$ \\
$\mathrm{L} 1 / 2$ & $5(13.2)$ & $5(10.4)$ & $2(18)$ \\
$\mathrm{L} 2 / 3$ & $3(7.9)$ & $4(8.3)$ & $0(0)$ \\
$\mathrm{L} 3 / 4$ & $2(5.3)$ & $2(4.2)$ & $2(18)$ \\
$\mathrm{L} 4 / 5$ & $1(2.6)$ & $0(0)$ & $1(9)$ \\
L5/S1 & $7(18.4)$ & $3(6.3)$ & $5(45.5)$ \\
\hline
\end{tabular}

NB. Degenerative disc disease (DDD), vertebral end changes (VEP) and disc herniations, $n=$ number of outcomes and some participants had multiple outcomes

spinal levels combined (Table 2). At the time of their imaging, $31.8 \%$ with DDD had current LBP, with $86.4 \%$ reporting a history of LBP.

\section{VEP changes}

Seven participants $(42.5 \%)$ demonstrated VEP changes (Table 1), with T11/12 the level most frequently affected (29.2\%) followed by T10/11 (22.9\%) (Table 2). The most frequent abnormalities were seen at the superior and inferior aspects of T11 and the superior aspect of T12. The MRI pattern of VEP changes included oedema adjacent to the end plate and a more chronic pattern of end plate irregularity and sclerosis. A notable observation was that participants who presented with DDD also exhibited VEP changes at the T10/11 and T11/12 levels (Table 2). 


\section{Disc herniation}

Eight participants (20\%) demonstrated 11 disc herniations (Table 1). Three participants had disc herniations at two levels with the majority of herniations (5/11) at the L5/ S1 level. Three of these were generalised disc bulges with the other three characterised as small central protrusions. Other affected levels included T12/L1 (1/11) and L1/2 (2/11) with 3 generalised disc bulges. Two participants had L3/4 involvement ( 1 central disc protrusion, 1 generalised disc bulge) with 1 participant with L4/5 central disc protrusion (Table 2). None of the disc herniations involved significant nerve root compression.

\section{Posterior element abnormalities}

Table 3 shows the distribution and location of spondylolysis and spondylolisthesis.

\section{Spondylolysis}

Pars interarticularis stress reactions were seen in 9 participants $(22.5 \%)$. Complete pars injuries (stress fracture) were evident in 7 participants (17.5\%), all at the L5 level.

\section{Spondylolisthesis}

Spondylolisthesis was present in 6 participants (15\%) all affecting the L5/S1 level. All 6 participants reported a history of LBP, and 2 participants reported current LBP. Bilateral L5 foraminal narrowing was documented in 3 participants.

Table 3 Distribution and location of spondylolysis and spondylolisthesis

$n=22$

$n(\%)$

Complete pars injuries

Chronic bilateral complete L5 pars defect

7 (31.8)

Stress reactions

Left L2 pedicle, pars and transverse process oedema

Mild left L3 pedicle oedema

Right L3 pars, lamina and transverse process oedema

Bilateral L3 pedicle and pars oedema $1(4.5)$

Right L4 posterior pedicle oedema $1(4.5)$

Bilateral L5 pars sclerosis, no defect 1 (4.5)

Bilateral posterior pedicel L5 oedema $1(4.5)$

Left L5 pars oedema 1 (4.5)

Left L5 pars and lamina oedema 1 (4.5)

Spondylolisthesis

L5/S1

$6(27.3)$

\section{Facet joint abnormality}

Facet joint arthropathy was demonstrated in 6 participants (15\%) occurring at L4/5 or L5/S1 levels. Two participants had bilateral facet joint effusions at L4/5 and L5/S1 with another having left L4/5 and bilateral L5/S1 effusions. One participant had a right L4/5 effusion, and one had a left L5/S1 facet joint cyst. Two participants with facet joint arthropathy also demonstrated DDD. One participant with L5/S1 facet joint degenerative changes also had disc height loss at T11/12, L1/2 and L5/S1 levels. One participant with a small facet joint effusion at L4/L5 and L5/S1 also demonstrated L5/S1 disc height loss.

\section{Other abnormalities}

A left L5 perineural cyst and one individual with a minor scoliosis in the mid-lumbar region were identified.

\section{Comparison of MRI positions (Objective 2)}

From 30 participants with abnormal scans, 23 had identical scans when imaged in positions of UFP and UEP, i.e. the dynamic examination did not reveal any additional findings. Seven participants demonstrated different MRI findings across imaging positions (5 females, aged 14-20 years). Only one participant had an alteration to disc morphology displaying a more prominent L5/S1 disc protrusion in UEP and less prominent in UFP.

Six participants had alterations to the posterior element abnormalities including the degree of fluid within the facet joints, spinal alignment secondary to the spondylolisthesis and the size of the pars defect. From these six, four participants had increased fluid in the facet joints in UFP. Pars injuries were clearer in two participants with one having an increase in anterolisthesis in the UFP and UEP compared to SS (Table 4).

Two other participants included one with a very small amount of fluid in the facet joint with a pars fracture that is difficult to appreciate in SS. An increase in the volume of fluid in the facet joint was seen, and the pars fracture became more clearly visible in UEP. In UFP, there was also an increase in the degree of facet joint fluid, but the pars fracture was not visible (Fig. 1a, b and c).

The other participant had DDD with loss of disc height and hydration, and a grade 1 spondylolisthesis, which clearly visualised in SS. In UFP, the degree of anterior translation was visible as a grade 2 spondylolisthesis (Fig. 2a and b).

\section{Discussion}

Findings from this prospective cross-sectional observational study evidence $75 \%$ demonstrated abnormality with a wide variety of altered disc morphology and posterior element 
Table 4 Differences in MRI findings documented across imaging positions $(n=7)$

\begin{tabular}{|c|c|c|c|}
\hline Participant & SS & UFP & UEP \\
\hline 1 & $\begin{array}{l}\text { Posterior element abnormality: } \\
\text { Chronic bilateral complete L5 pars defects } \\
\text { Gr1 L5/S1 spondylolisthesis }\end{array}$ & \multicolumn{2}{|c|}{ No change in anterolisthesis, increased fluid in L5/S1 facet joints } \\
\hline 2 & $\begin{array}{l}\text { Posterior element abnormality: } \\
\text { Chronic bilateral complete L5 pars defects } \\
\text { with Gr1 L5/S1 spondylolisthesis }\end{array}$ & \multicolumn{2}{|l|}{$\begin{array}{l}\text { No change in anterolisthesis or facet joints } \\
\text { Pars defect better seen }\end{array}$} \\
\hline 3 & $\begin{array}{l}\text { Posterior element abnormality: } \\
\text { Chronic bilateral complete L5 pars defects } \\
\text { Gr1 L5/S1 spondylolisthesis } \\
\text { Bilateral L5 foraminal narrowing }\end{array}$ & \multicolumn{2}{|l|}{ Increased anterolisthesis compared to neutral } \\
\hline 4 & $\begin{array}{l}\text { Posterior element abnormality: } \\
\text { Chronic bilateral complete L5 pars defects } \\
\text { Gr1 L5/S1 spondylolisthesis } \\
\text { Small facet joint effusions at L4/L5, L5/S1 }\end{array}$ & $\begin{array}{l}\text { Pars defects better seen, reduced foraminal } \\
\text { compromise, no change in anterolisthesis, } \\
\text { fluid in L4-5 facet joints increased in } \\
\text { flexion }\end{array}$ & $\begin{array}{l}\text { No change in anterolisthesis, increased } \\
\text { foraminal narrowing }\end{array}$ \\
\hline 5 & $\begin{array}{l}\text { Altered disc pathology: } \\
\text { T12/L1 and L5/S1 disc height loss } \\
\text { L5/S1 central disc protrusion } \\
\text { VEP changes at inferior T12 and superior } \\
\text { L1 }\end{array}$ & \multicolumn{2}{|l|}{$\mathrm{L} 5 / \mathrm{S} 1$ disc protrusion less prominent } \\
\hline 6 & $\begin{array}{l}\text { Posterior element abnormality: } \\
\text { Bilateral facet joint effusions at L5/S1 and } \\
\text { left sided L4/5 } \\
\text { Chronic bilateral complete L5 pars defects } \\
\text { Gr1 L5/S1 spondylolisthesis }\end{array}$ & $\begin{array}{l}\text { Increased facet joint fluid L4/5 and L5/ } \\
\mathrm{S} 1 \text {, pars defects no change, no change in } \\
\text { anterolisthesis }\end{array}$ & $\begin{array}{l}\text { Pars defects more clearly visualised, } \\
\text { increased L5/S1 facet fluid bilaterally }\end{array}$ \\
\hline 7 & $\begin{array}{l}\text { Posterior element abnormality: } \\
\text { Chronic bilateral complete L5 pars defects } \\
\text { Gr1 L5/S1 spondylolisthesis with bilateral } \\
\text { L5 foraminal narrowing } \\
\text { Bilateral facet joint effusions L4/5 and L5/ } \\
\text { S1 }\end{array}$ & Increased L5/S1 facet fluid & $\begin{array}{l}\text { Increased L5/S1 fluid bilaterally, pars } \\
\text { defects more clearly visualised }\end{array}$ \\
\hline
\end{tabular}

abnormality in the thoracolumbar spine of elite gymnasts. With fifteen participants $(37.5 \%)$ reporting current LBP and thirty-three $(82.5 \%)$ reporting a history of LBP investigations identifying early signs of abnormality will add value to long-term management.

\section{Altered disc morphology}

Findings that $55 \%$ of participants exhibited DDD are consistent with some existing evidence [3], although higher than some [18] and lower than others [1]. The greater prevalence of DDD within our study may be explained by the increased training loads, skill level and complexity of routines at the elite level inducing greater load on the spine versus collegiate-level gymnasts [18]. Unlike previous studies [1, 3] multi-level disc disease was reported, with almost a third having two involved levels and a fifth having single-level involvement.

The most frequent level of DDD was T11/12, a higher anatomical level than previous evidence [19] and not at the expected lumbosacral junction. A key finding was that DDD and VEP changes were seen at T10/11, T11/12 suggesting the thoracolumbar junction is a transition zone during the extreme motions, second to the stress phenomena [20]. Our study supports the hypothesis from Murphy et al. (2019) that thoracolumbar transition is higher (at T10-11) than expected.

Morphological changes to the VEP are normally seen in advancing age groups but are also apparent in association with pathological changes to the nucleus and annulus in advanced stages of DDD [21]. The recent findings suggest that disc degeneration in elite gymnasts follows a similar pattern of degeneration, suggesting repeated compression and flexion stresses on the lumbar spine may contribute. The stress injury mechanism with loss of disc height could consequently increase stress on the posterior element [22], all of which occur during the repetitive multi-directional spinal loading in gymnastics training.

\section{Posterior element abnormality}

Athletic populations are predisposed to posterior element abnormalities, and our study showed that gymnasts had pars oedema at higher rates $(40 \%)$ compared to other sports including diving (35.38\%) and rugby (22.22\%) [23]. Within our study, $15 \%$ had spondylolisthesis, all affecting the L5/S1. 


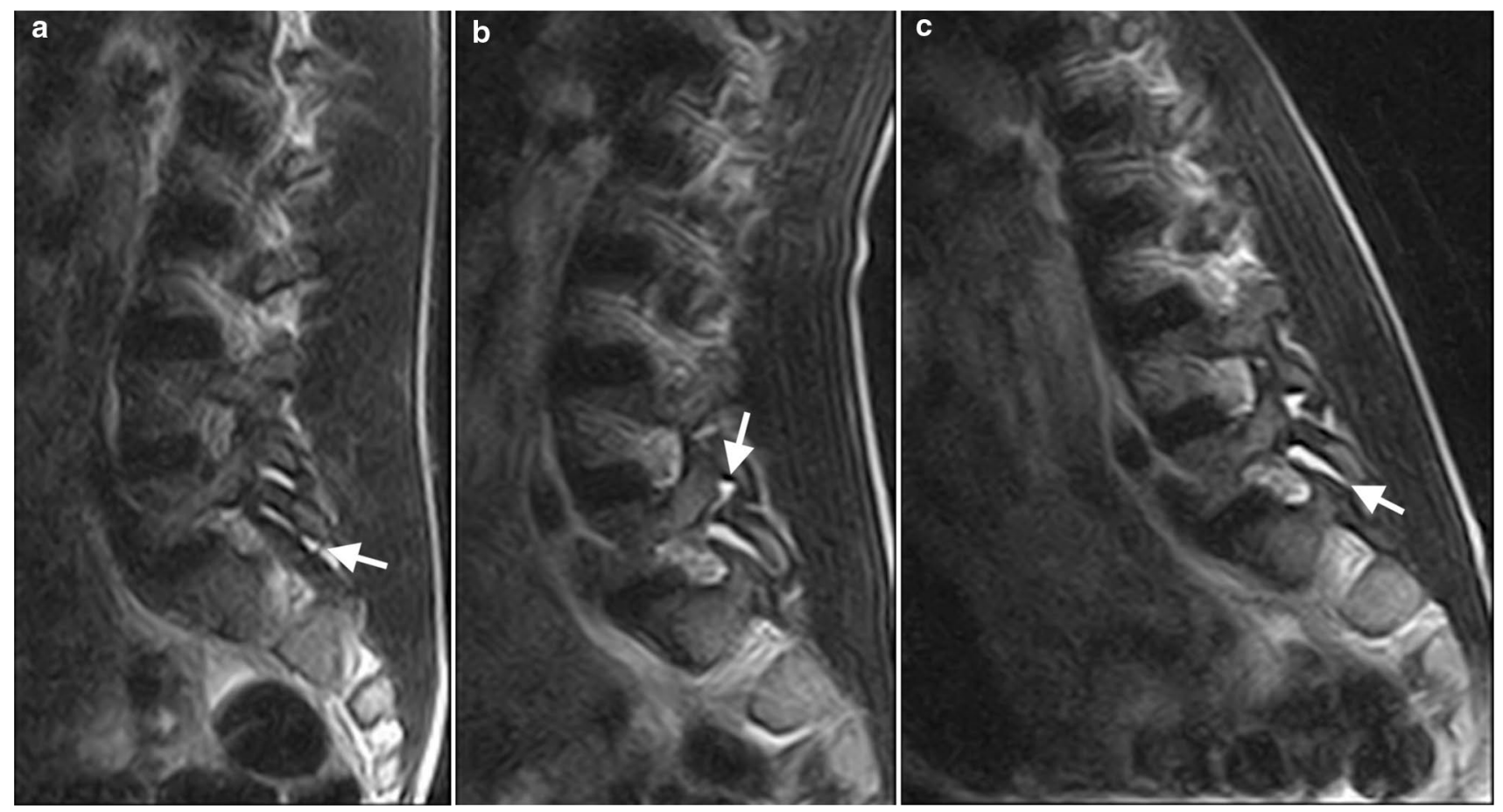

Fig. 1 a Parasagittal T2-weighted sequence in neutral. There is a very small amount of fluid in the facet joint (white arrow), and the pars fracture is difficult to appreciate. b Parasagittal T2-weighted sequence in extension. There has been an increase in the volume of fluid in the facet joint, and the pars fracture is now clearly seen (white arrow). c Parasagittal T2-weighted sequence in flexion. There has been an increase in the degree of fluid in the facet joint, but the pars fracture is not visualised
Fig. 2 a Parasagittal

$\mathrm{T} 2$-weighted sequence in neutral. There is disc degeneration with loss of disc height and hydration. There is a grade 1 spondylolisthesis (white arrow). b Parasagittal T2-weighted sequence in flexion. There has been an increase in the degree of anterior translation in flexion with a grade 2 spondylolisthesis now demonstrated (white arrow)
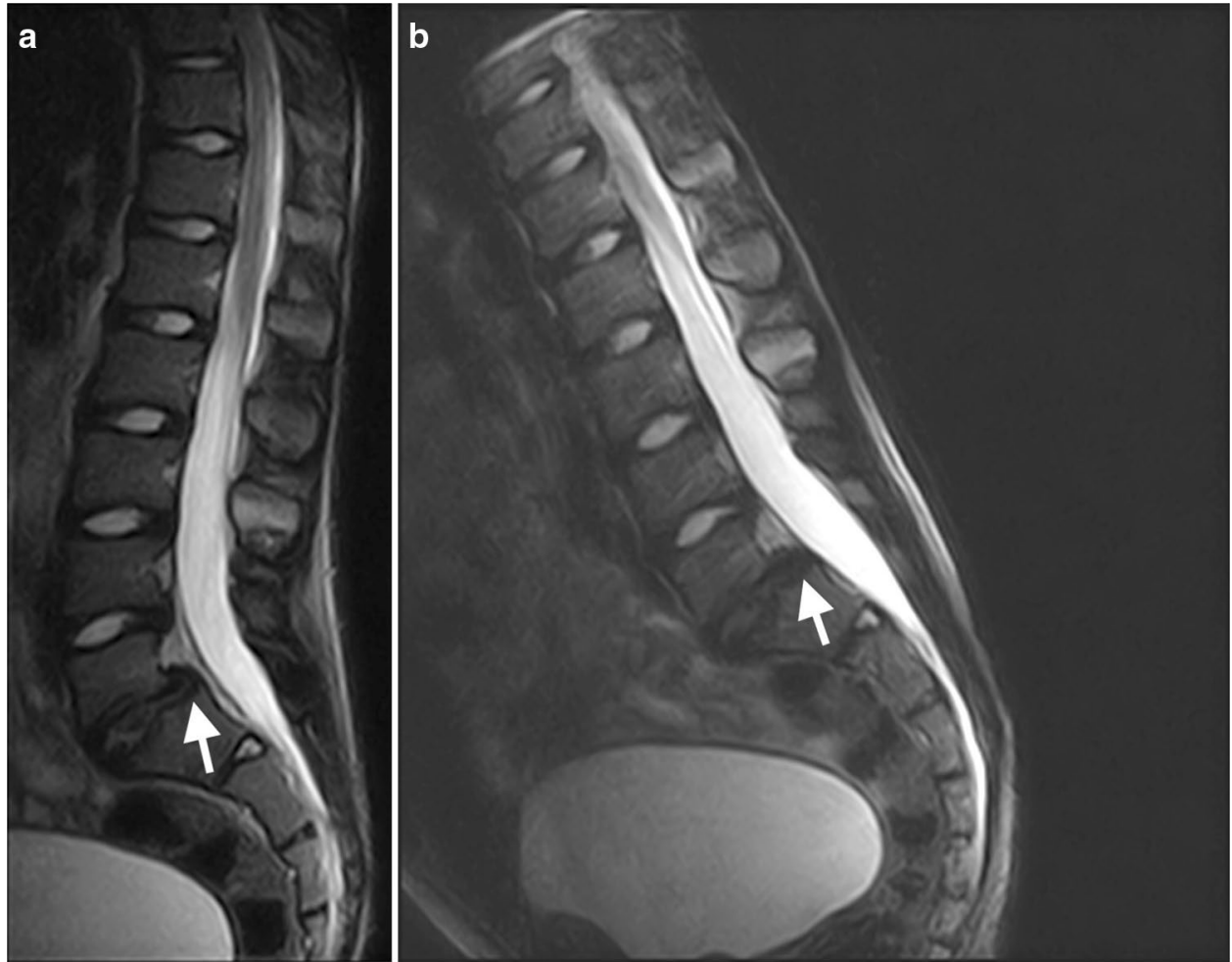
Previous research [5] has shown findings of spondylolysis, spondylolisthesis, bilateral bone marrow oedema were only seen in symptomatic participants which is different to this study.

Research supports that the growing spine is highly vulnerable to trauma, especially during the adolescent growth spurt [24]. Ages of the gymnasts with spondylolisthesis were between 14 and 17 (mean 15.2), with a ratio of 2:1 female to male. Training loads and overuse injury problems are reported as high within young age groups suggesting long training duration as a source [25], and our results support this with reported mean training hours as 32 (SD: 5.3) hours per week. Artistic gymnastics is classed as an early specialisation sport, with intensive athlete involvement at an early age including participation and competition, characterised with an early focus on performance improvement and success. Clinical practice, coaching methods and athlete's careers could all benefit from this greater understanding of the pathologies present, but further insight is needed into the clinical presentation and follow-up into long-term effects.

\section{Comparison of MRI positions}

Seven out of 30 (23\%) participants with abnormal scans demonstrated different MRI findings according to imaging position with 6 showing alterations to posterior element abnormalities.

Pars defects and spondylolisthesis were visible in SS, and there were no new cases where pars defects or additional spondylolisthesis was found on UFP or UEP. When present, it was reported that spondylolysis defects were more readily appreciated on MRI in SS. Most cases showed stable spondylolisthesis, i.e. a slip but not "mobile", and it is also unlikely that there was dynamic nerve root compression. UFP and UEP led to altered loading of facet joints, so alterations to fluid distribution within the joint were evident. These findings are unlikely to be significant clinically, but this altered loading may explain why pars defects were better appreciated. This study demonstrates that upright MRI could provide key information to establish the extent of early imaging abnormalities.

\section{Strengths and limitations}

Small numbers in our study may be perceived as a limitation, but recruiting a large relatively homogenous group of elite gymnasts is difficult. A higher sample size would enable greater exploration of the impact of age, gender and training load. Inter-observer reliability was not assessed, but independent assessment of imaging from experienced radiologists validates findings, and the blinding of the radiologists is a strength.

\section{Conclusion}

Findings suggest high levels of MRI abnormalities in elite gymnastics including altered disc morphology and posterior element abnormalities. High prevalence of T11/12 DDD and VEP changes reflects the thoracolumbar junction being a transition zone during the extreme ranges required in gymnastics which may influence future management and prevention strategies especially during spinal maturation. Upright MRI and varying spine position offer promise for enhanced visualisation of posterior element abnormalities. This is extremely important in the athletic population demonstrating value of MRI in different positions to justify further research.

\section{Appendix 1}

\section{What are the new findings?}

\section{How might it impact on clinical practice in the future?}

- Seventy-five per cent of gymnasts had MRI abnormalities in the
thoracolumbar spine with 55\% showing evidence of altered disc
morphology and $40 \%$ showing posterior element abnormalities
- DDD was most evident in 55\% participants, the majority at T11/12.
VEP changes were evident in $42.5 \%$ mostly at T11/12
- Spondylolysis was present in $40 \%$ with an additional $17 \%$ showing
chronic bilateral complete L5 pars defects, and 15\% of these having
grade 1 L5/S1 spondylolisthesis
- $23 \%$ demonstrated different MRI findings when placed in upright
flexion and extension, predominantly resulting in reporting amend-
ments indicating a greater extent of posterior element abnormality
which is new evidence in gymnasts

- It is important to understand the prevalence of imaging abnormalities in asymptomatic populations, and this study will assist clinicians and patients interpret MRI findings in the context of the clinical presentation

- A higher level of pathology at the thoracolumbar junction and not at the expected lumbosacral junction could influence future management and prevention strategies

- The first study in elite gymnastics to explore upright MRI justifies further research 
Acknowledgements To the participants of this study who shared their time. To British Gymnastics and the English Institute of Sport who provided endorsement of this study. To Jason Laird (Lead Physiotherapist at British Gymnastics) for his contribution to this study.

Authors' contributions LF, AR and SJ created and designed the study. LF, SJ, RB, AR and NH analysed and interpreted the data. All authors contributed to the development of the manuscript.

Funding This work was funded by a research grant from the British Society of Skeletal Radiologists and the Musculoskeletal Association of Chartered Physiotherapists. Both providers were not involved in the research process. The lead author's $\mathrm{PhD}$ tuition fees are funded by the English Institute of Sport.

Availability of data and material Available on request from the corresponding author.

\section{Declarations}

Conflict of interest The authors declare that they have no conflict of interest.

Consent for publication All authors consent to publication.

Ethical approval The University of Birmingham Research Ethics committee (id.ERN_16-0269).

Consent to participate All participants agreed to consent.

Open Access This article is licensed under a Creative Commons Attribution 4.0 International License, which permits use, sharing, adaptation, distribution and reproduction in any medium or format, as long as you give appropriate credit to the original author(s) and the source, provide a link to the Creative Commons licence, and indicate if changes were made. The images or other third party material in this article are included in the article's Creative Commons licence, unless indicated otherwise in a credit line to the material. If material is not included in the article's Creative Commons licence and your intended use is not permitted by statutory regulation or exceeds the permitted use, you will need to obtain permission directly from the copyright holder. To view a copy of this licence, visit http://creativecommons.org/licenses/by/4.0/.

\section{References}

1. Sward L, Hellstrom M, Jacobsson B et al (1990) Back pain and radiologic changes in the thoraco-lumbar spine of athletes. Spine $15: 124-129$

2. Wade M, Campbell A, Smith A et al (2012) Investigations of spinal posture signatures and ground reaction forces during landing in elite female gymnasts. J Appl Biomech 28:677-686

3. Bennett DL, Nassar L, DeLano MC (2006) Lumbar spine MRI in the elite-level female gymnast with low back pain. Skelet Radiol 35:503-509

4. Roberts S (2002) Disc morphology in health and disease. Biochem Soc Trans 30:864-869

5. Hellström M, Jacobsson B, Swärd L et al (1990) Radiologic abnormalities of the thoraco-lumbar spine in athletes. Acta Radiol $31: 127-132$

6. Motley G, Nyland J, Jacobs J et al (1998) The pars interarticularis stress reaction, spondylolysis, and spondylolisthesis progression. J Athl Train 33:351-358
7. Ranson CA, Kerslake RW, Burnett AF et al (2005) Magnetic resonance imaging of the lumbar spine in asymptomatic professional fast bowlers in cricket. J Bone Jt Surg 87:1111-1116

8. Goldstein JD, Berger PE, Windler GE et al (1991) Spine injuries in gymnasts and swimmers. An epidemiological investigation. Am J Sports Med 19:463-468

9. Mohriak R, Silva PDV, Trandafilov M et al (2010) Spondylolysis and spondylolisthesis in young gymnasts. Rev Bras Ortop 45:79-83

10. Alyas AB, Connell D, Saifuddin AB (2008) Upright positional MRI of the lumbar spine F. Clin Radiol 63:1035-1048

11. von Elm E, Altman DG, Egger W et al (2014) The strengthening the reporting of observational studies in epidemiology (STROBE) statement: guidelines for reporting observational studies. Int J Surg 12:1495-1499

12. Thompson C (1999) If you could just provide me with a sample: examining sampling in qualitative and quantitative research papers. Evidenced Based Nurs 2:68-70

13. Pfirrmann CW, Metzdorf A, Zanetti M et al (2001) Magnetic resonance classification of lumbar intervertebral disc degeneration. Spine 26:1873-1878

14. Farndon DF, Milette PC (2001) Nomenclature and classification of lumbar disc pathology. Recommendations of the combined task forces of the North American spine society, American society of spine radiology, and American society of neuroradiology. Spine 26:E93-E113

15. Modic MT, Masaryk TJ, Ross JS et al (1988) Imaging of degenerative disk disease. Radiology 168:177-186

16. Hollenburg G, Beattie P, Meyers S et al (2002) Stress reactions of the lumbar pars interarticularis: the development of new MRI classification system. Spine 27:181-186

17. Weishaupt D, Zanetti M, Boos N et al (1999) MR Imaging and CT in osteoarthritis of the lumbar facet joints. Skel Radiol 28:215-219

18. Koyama K, Nakazato K, Min SK et al (2013) Radiological abnormalities and low back pain in gymnasts. Int J Sports Med 34:218-222

19. Pouriesa M, Fouladi RF, Mesbahi S (2013) Disproportion of end plates and the lumbar intervertebral disc herniation. Spine $\mathrm{J}$ 13:402-407

20. Murphy J, McLoughlin E, Davies AM et al (2020) Is T9-11 the true thoracolumbar transition zone? J Clin Orthop Trauma 11:891-895

21. Vernon-Roberts B (1992) Age -related and degenerative pathology of intervertebral discs and apophyseal joints. In: Jayson MIV (ed) The lumbar spine and back pain, 4th edn. Churchill Livingstone, Edinburgh, pp 17-41

22. Latham JM, Pearcy MJ, Costi JJ et al (1994) Mechanical consequences of annular tears and subsequent intervertebral disc degeneration. Clin Biomech 9:211-219

23. Tawfik S, Phan K, Mobbs R (2020) The incidence of pars interarticularis defects in athletes global. Spine J 10(1):89-101

24. Alexander J (1976) Effect of growth rate on the strength of the growth plate-shaft junction. Skelet Radiol 1:67-76

25. Kujala UM, Taimela S, Erkintalo M et al (1996) Low-back pain in adolescent athletes. Med Sci Sports Exerc 28:165-170

Publisher's Note Springer Nature remains neutral with regard to jurisdictional claims in published maps and institutional affiliations. 